epidemic drivers, the Transmission Dynamics Epidemic Classification (TDEC) scheme, and demonstrated its application. Where data permitted, we calculated the population attributable fraction of paid sex for HIV infection among males to assist in TDEC classification.

Results Country and district level analysis illustrated three main limitations of the numeric and MOT methods: (1) their results misinterpreted underlying transmission dynamics and were inconsistent; (2) they were difficult to apply to local epidemics with heterogeneity across districts; and (3) the MOT model was highly sensitive to input parameters, many of which required extraction from non-regional sources. The TDEC method offered a logical algorithm to characterise local sexual structures that likely sustain onward HIV transmission; it required minimal but key input data. Conclusion Traditional appraisals of HIV epidemics can misdirect prevention programming if the goal is long-term HIV control. By characterising local transmission dynamics, the TDEC approach provides a potentially more effective tool with which policy makers can design intervention programs.

\section{P1-S4.16 THE UNAIDS MODES OF TRANSMISSION MODEL: A USEFUL TOOL FOR DECISION MAKING?}

doi:10.1136/sextrans-2011-050108.160

${ }^{1} \mathrm{~A}$ Foss, ${ }^{1} \mathrm{H}$ Prudden, ${ }^{1} \mathrm{~A}$ Mehl, ${ }^{1} \mathrm{C}$ Zimmerman, ${ }^{2} \mathrm{~K}$ Ashburn, ${ }^{2} \mathrm{R}$ Trasi, ${ }^{3} \mathrm{M}$ Kiess, ${ }^{4} \mathrm{G}$ Bantebya-Kyomuhendo, ${ }^{2} \mathrm{~K}$ Fritz, ${ }^{1} \mathrm{C}$ Watts. ${ }^{1}$ London School of Hygiene \& Tropical Medicine, London, UK; ${ }^{2}$ International Center for Research on Women, Washington, DC, USA; ${ }^{3}$ International Center for Research on Women-Cambodia, Phnom Penh, Cambodia; ${ }^{4}$ Makerere University, Kampala, Uganda

Background The UNAIDS Modes of Transmission (UNAIDSMoT) model is increasingly being used to inform national-level HIV priority setting. Although the model is simple to use, important factors may be omitted. Using the UNAIDSMoT model, with Uganda and Cambodia as case studies, we explored the influence of the incorporation of additional factors.

Methods A detailed review of the UNAIDSMoT model was conducted, including reviewing the model structure, data requirements, parameters and assumptions. Country-specific literature reviews were undertaken, along with field visits involving keyinformant interviews, to identify the main features of the HIV epidemic in each setting. Adaptations were made to the
UNAIDSMoT model to explore the sensitivity of the model projections to these revisions.

Results A key limitation of the original UNAIDSMoT model is that it does not allow an individual's multiple sources of risk to be considered. The model was adapted to incorporate important multiple risk sources (including injecting drug use), and the effects of the 2008 change in Cambodian law on human trafficking which led to brothel closures, the migration of many sex workers into entertainment venues, and the discontinuation of numerous outreach and condom distribution programmes. As illustrated in Abstract P1-S4.16 figure 1, the revised model projects lower percentages of new HIV infections occurring among sex workers ( $2 \%$ vs $5 \%$ ) and the regular female partners of men who buy sex ( $5 \%$ vs $48 \%$ ) than the original model, primarily replaced with new infections occurring in the new groups: entertainment workers who sell sex (38\%), and injecting drug users and their sexual partners (5\%). In Uganda, the high prevalence of HIV infection among women means that the static model predicts men are more at risk of infection from their female partners than women are from their male partners. This fails to reflect the dynamic chain of HIV transmission.

Conclusions Our analysis highlights the limitations of the UNAIDSMoT model, and the potential dangers of over-interpretation of a relatively simplistic and static reflection of a dynamic HIV epidemic (and prevention priorities) at a particular time-point. It is important to regularly re-structure and re-parameterise models according to new data and changes in patterns of risk behaviours. Further research to test the validity, sensitivity and robustness of the UNAIDSMoT model projections in different epidemic settings is needed.

\section{P1-S4.17 INTERACTIVE DEMONSTRATION OF A MODEL TO CALCULATE COSTS AND COST EFFECTIVENESS OF DIFFERENT STRATEGIES FOR CHLAMYDIA SCREENING AND PARTNER NOTIFICATION}

doi:10.1136/sextrans-2011-050108.161

${ }^{1} \mathrm{~K}$ Turner, ${ }^{2} \mathrm{E}$ Adams, ${ }^{3} \mathrm{~A}$ Grant, ${ }^{1} \mathrm{~J}$ Macleod, ${ }^{4} \mathrm{G}$ Bell, ${ }^{5} \mathrm{~J}$ Clarke, ${ }^{6} \mathrm{P}$ Horner. ${ }^{1}$ University of Bristol, Bristol, UK; ${ }^{2}$ Independent, London, UK; ${ }^{3}$ Pathway Analytics, UK; ${ }^{4}$ Royal Hallamshire Hospital, UK; ${ }^{5}$ Teaching Hospital, University of Leeds, UK; ${ }^{6}$ University of Bristol, UK

Background We have developed a model to calculate costs and cost effectiveness of different intervention strategies for chlamydia

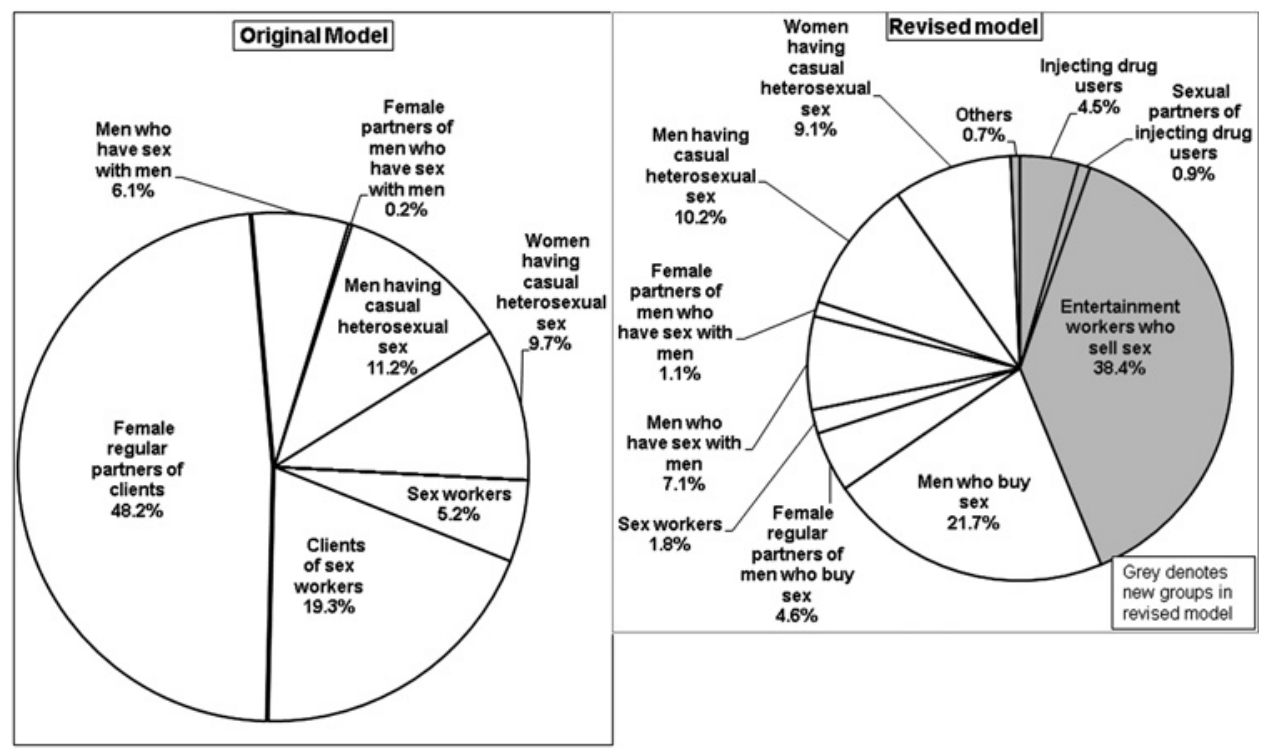

Abstract P1-S4.16 Figure 1 Distribution of HIV incidence in original and revised UNAIDSMoT models. 
screening, which can be used by commissioners to evaluate and plan their local service provision. The model and an analysis of national data have been recently published in BMJ. Increasingly, financial constraints will mean that tools such as ours will be vital to provide value for money in healthcare.

Method We will give a live demonstration of our spreadsheet model using the example from our recent study. We invite delegates to input their own data in real time to a laptop for comparison and testing against the national and international picture. We plan to promote this before the meeting.

Main outcome measures Cost effectiveness of screening (as cost per individual tested, cost per positive diagnosis, total cost of screening, number screened, number infected, sex ratio of those tested and treated). We will show how to compare current programmes with a planned change eg, in screening coverage or partner notification efficacy. Results In 2008-2009 screening was estimated to cost $£ 46.3 \mathrm{~m}$ and $£ 506$ per infection treated. The model results suggest that increasing male screening coverage from $8 \%$ to $24 \%$ (to match female coverage) would cost an extra $£ 22.9 \mathrm{~m}$ and increase the cost per infection treated to £528. Increasing partner notification efficacy from 0.4 to 0.8 partners per index case would cost an extra $£ 3.3 \mathrm{~m}$ and reduce the cost per infection diagnosed to $£ 449$.

Conclusions The results are broadly representative of the national picture, but further evaluation of the cost effectiveness of partner notification and screening is urgently needed. We encourage practitioners who want to test this tool in their own setting to contact us (katy.turner@bristol.ac.uk).BMJ 2011; 342:c7250 10.1136/bmj.c7250.

\section{P1-S4.18 USING MATHEMATICAL MODELLING TO INVESTIGATE THE ROLE OF THE HIDDEN “POPULATION OF MEN WHO HAVE SEX WITH MEN (MSM) ON THE HIV EPIDEMIC IN SOUTHERN INDIA"}

doi:10.1136/sextrans-2011-050108.162

${ }^{1} \mathrm{H}$ Prudden, ${ }^{1} \mathrm{~A}$ Foss, ${ }^{1} \mathrm{~K}$ Mitchell, ${ }^{2} \mathrm{M}$ Pickles, ${ }^{2} \mathrm{~A}$ Phillips, ${ }^{3,4} \mathrm{~B} \mathrm{M}$ Ramesh, ${ }^{3,4,5} \mathrm{R}$ Washington, ${ }^{6} \mathrm{M}$ Alary, ${ }^{7} \mathrm{C}$ Lowndes, ${ }^{1} \mathrm{P}$ Vickerman. ${ }^{1}$ London School of Hygiene and Tropical Medicine, London, UK; ${ }^{2}$ Imperial College London, London, UK; ${ }^{3}$ Karnataka Health Promotion Trust, Bangalore, India and; " University of Manitoba Winnipeg, Canada; ${ }^{5}$ St John's Research Institute, Bangalore, India; ${ }^{6}$ Centre hospitalier affilié universitaire de Québec, Québec, Canada; ' Health Protection Agency, London, UK Canada

Background Biological and behavioural data for men who have sex with men (MSM) in Bangalore, Karnataka, India, have mainly been collected from sites where commercial sex is prevalent. Consequently, the survey data may fail to capture the behaviour of a larger lower-risk "hidden" MSM population. Mathematical modelling is used to explore the potential bias in the survey data and better quantify the characteristics of this hidden population.

Methods A dynamic model of HIV transmission among MSM was developed and parameterised using detailed data* from high risk MSM in urban Bangalore. The MSM were categorised into three subgroups: Kothi and Hijra(KH): who mostly take the receptive role in anal sex, Panthis and Bisexuals(PB) who are predominantly insertive partners and Double Deckers(DD) who take both roles. Due to the sampling methods used, it was thought the MSM survey data were more representative of $\mathrm{KH}$ and $\mathrm{DD}$ than $\mathrm{PB}$, although the extent of this bias is unknown. Therefore, no fitting constraint was applied to the PB HIV prevalence and instead the model was used to explore what PB HIV prevalence values are projected if the model was only fit to the $95 \%$ CIs of the prevalence data for $\mathrm{KH}$ and DD. One million randomly sampled model simulations were undertaken to find model fits.

Results Abstract P1-S4.18 figure 1 shows that, although the model can produce HIV prevalence estimates consistent with the survey estimates, overall the model projections suggest a lower PB HIV prevalence is more consistent with the survey estimates for $\mathrm{KH}$ and DD. In addition, $80 \%$ of the model fits to the $\mathrm{KH}$ and DD HIV prevalence data had a sampled frequency of sex acts for $\mathrm{PB}$ in the lower half of the uncertainty interval suggesting PB's sexual activity may be lower than the median reported in the MSM survey. As expected, an inverse relationship occurs between $\mathrm{PB}$ population size and their level of sexual activity, with the median $\mathrm{PB}$ population size being 55400 (2.7\% of the total urban male population) and varying between 28000 and 73000 (1.5 to 3.9\% of the total urban male population) for the IOR of the model fits Conclusions: Survey data imply MSM are a small, highly active group, many of whom regularly sell sex, have very high numbers of partners and typically take the receptive role. As demonstrated here, modelling can be used to provide insights into the likely HIV prevalence, population size and sexual activity of hidden "MSM not reached in surveys."

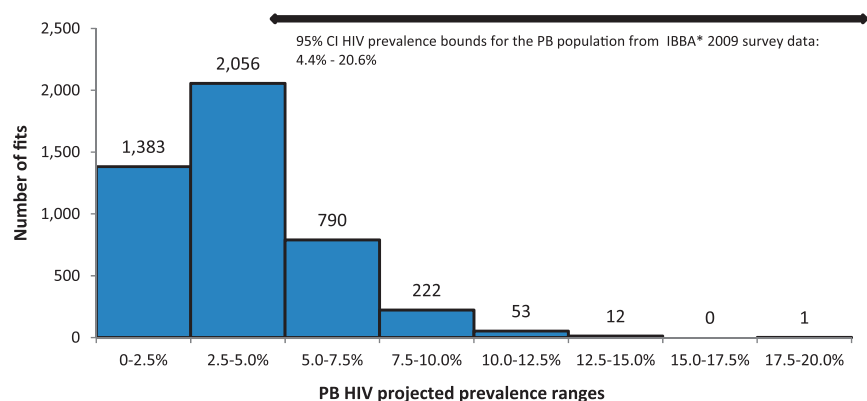

Abstract P1-S4.18 Figure 1 Number and range of projected HIV prevalence estimates for the PB population (from the model fits to $\mathrm{KH}$ and DD data. * Integratedbiological and behavioural assessment (IBBA) 2009 , collected within the monitoring and evaluation of Avahan, the India AIDS initiative.

\section{P1-S4.19 STOCHASTIC NETWORK MODELS}

doi:10.1136/sextrans-2011-050108.163

P A Noël, A Allard, L Hébert-Dufresne, V Marceau, L J. Dubé. Université Laval, Québec, Canada

Background We present a general modelling scheme particularly adapted for the epidemiology of sexually transmitted infections. Emphasis is placed on the complex interaction structure of the population and on the probabilistic nature of the dynamics.

Methods We represent the interaction structure of the population with complex network models. Markov Stochastic Processes are used to consider the probabilistic time evolution of both the network structure and the epidemiological state of the population. Idealised epidemiological problems are considered: they are qualitatively inspired by real-world systems but no actual real-world data is fed to the models. All analytical results are systematically validated through Monte Carlo numerical simulations.

Results By dedicating compartments to individuals with both similar epidemiological states and similar contact patterns, we observe great agreement between analytical results and Monte Carlo simulations see Abstract P1-S4.19 Figure 1. This is a special case of the general observation that compartmental models perform better when individuals within each compartment are very similar among themselves. Explicit examples are given for contact patterns changing through time as well as for the interactions of different infections in the same population.

Conclusions By specifying the structure of the population through its local features, we successfully model the probabilistic evolution of complex epidemiological systems. Infection stages and/or behavioural groups are considered the same way they are in "classical" compartmental models. The generality of the approach facilitates its application to a vast array of epidemiological phenomena. 